\title{
Comparison of Auditory Evoked Potentials in Heterosexual, Homosexual, and Bisexual Males and Females
}

\author{
Dennis McFadden and Craig A. Champlin \\ Departments of Psychology and Communication Sciences and Disorders and the Institute for Neuroscience, \\ University of Texas, Austin, TX 78712 USA
}

Received: 17 December 1999; Accepted: 30 March 2000; Online publication: 13 July 2000

\begin{abstract}
The auditory evoked potentials (AEPs) elicited by click stimuli were measured in heterosexual, homosexual, and bisexual males and females having normal hearing sensitivity. Estimates of latency and/or amplitude were extracted for nine peaks having latencies of about 2$240 \mathrm{~ms}$, which are presumed to correspond to populations of neurons located from the auditory nerve through auditory cortex. For five of the 19 measures obtained, the mean latency or amplitude for the 57 homosexual and bisexual females was different from that of the 49 heterosexual females in a manner that implies a masculinization of the auditory systems of the homosexual and bisexual females. A similar masculinization effect was previously observed for the otoacoustic emissions generated by the cochlea. For five other measures, the mean latency or amplitude for the 53 homosexual and bisexual males was different from that of the 50 heterosexual males in a manner that implies a hypermasculinization of the auditory systems of the homosexual and bisexual males. Hypermasculinization has been reported recently for other physical characteristics of homosexual males. One parsimonious interpretation of these findings is that homosexual males and females both were exposed to higher than normal levels of androgens at some point(s) in development. Data are reported only for the female subjects not using oral contraceptives because those drugs can masculinize certain AEP measures.
\end{abstract}

Correspondence to: Dr. Dennis McFadden • Department of Psychology - Mezes Hall 330 - University of Texas - Austin, TX 78712-1189. Telephone: (512) 471-4324; fax: (512) 471-5935; e-mail: mcfadden@psy.utexas.edu
Keywords: auditory evoked potentials, homosexuality, masculinization, hypermasculinization, androgens, human prenatal development

\section{INTRODUCTION}

Two recent reports revealed that the otoacoustic emissions (OAEs) of homosexual and bisexual females were intermediate in number and strength to the OAEs of heterosexual females and heterosexual males (McFadden and Pasanen 1998, 1999). OAEs are sounds produced in the cochlea and emitted back through the middle ear into the external ear canal, where they can be detected using small microphone systems (Kemp 1978; reviewed by Probst et al. 1991). OAEs are more numerous and stronger in females than males (Talmadge et al. 1993; McFadden and Pasanen 1998, 1999). Those sex differences exist in infants and children as well as in adults (Burns et al. 1992; Norton 1992), and OAEs appear to be reasonably constant throughout life (Franklin et al. 1992; Burns et al. 1994). Taken together, the available evidence suggests that the sex differences in OAEs are determined by differential exposure to androgens during prenatal development (for the argument, see McFadden and Pasanen 1998, 1999). If one assumes that the choice of sex partners by female homosexuals is attributable to the masculinization of some brain structure(s) responsible for mate selection (Phoenix et al. 1959; Ellis and Ames 1987; Byne and Parsons 1993), then the implication is that those brain structures, and certain structures in the cochlea, were all masculinized as part of the same process. If this explanation is correct, one might expect to see evidence of this masculinization process in other sexually dimorphic measures. 
Human males and females differ on a number of auditory measures in addition to OAEs (McFadden 1998). One objective, physiological measure evidencing sex differences is auditory evoked potentials (AEPs). AEPs are gross potentials evoked by brief acoustic stimuli and measured using scalp electrodes and averaging techniques (Hall 1992). The result is a series of peaks or waves that are presumed to represent different populations of neurons firing more or less synchronously in response to the sound. When the latency or the amplitude is measured for these peaks, it is common to find differences between the sexes, which can persist even when the sex difference in head size is taken into account (Trune et al. 1988; Don et al. 1993). The existence of clear sex differences in certain AEP measures suggested that AEPs might also exhibit differences between heterosexuals and homosexuals. Measured in this experiment were latencies and amplitudes of nine peaks that constitute the auditory brainstem response (ABR), middle-latency response (MLR), and long-latency response (LLR).

\section{METHODS}

Subjects were recruited through local advertisements and by word of mouth. Prospective subjects were called for scheduling by a female lab assistant who explained the basic requirements of the experiment and emphasized that anyone exposed to intense sound or certain prescription, over-the-counter, and recreational drugs within the 24 hours immediately preceding the test session would not be included in the experiment. Test sessions for prospective female subjects not using oral contraception (OC) were scheduled for a time during days $16-26$ of the cycle because the ABR is known to fluctuate with the menstrual cycle (Elkind-Hirsch et al. 1992, 1994). Users of OC were tested without regard to the menstrual cycle, but the data for those subjects were excluded here after the discovery that OC masculinizes AEPs slightly (McFadden 2000). [The data reported here and in McFadden (2000) are from the same experiment.] Subjects were paid $\$ 30$ for about two hours work.

A test session involved measurements of three types-audiometric, psychological, and electrophysiological-conducted in that order after the subject had read and signed a consent form. Subjects revealing that they had forgotten the requirements of the experiment and had been exposed to intense sound or drugs in the previous 24 hours were dismissed for that day and rescheduled.

The audiometric screening established that each included subject had ear canals free from obstruction, normal tympanic-membrane mobility, and hearing sensitivity of $15 \mathrm{~dB}$ HL (Hearing Level) or better at octave frequencies between 250 and $4000 \mathrm{~Hz}$ plus 6000 $\mathrm{Hz}$ in both ears. Subjects passing the audiometric screening were given two timed tests and a questionnaire to complete. The timed tests assessed the ability to mentally rotate objects in space (Vandenberg and Kuse 1978) and to judge the level of fluid in vessels tilted off the vertical (Thomas et al. 1973). Those results will be reported elsewhere. The questionnaire contained items about various physical characteristics, historical patterns of exposure to intense sounds and drugs, and various aspects of sexual behavior.

Following completion of the questionnaire, the subject made a trip to the bathroom, and then was fitted with four gold-plated surface electrodes. One electrode was placed on the vertex $(\mathrm{Cz})$, one on the forehead (Fpz), and one on each earlobe (A1 and A2), using standard electroencephalographic techniques. The experimenter stressed the need for the subject to remain awake and to minimize eye blinks during the approximately $90 \mathrm{~min}$ of electrophysiological measurements. Wakefulness was monitored using the ongoing electroencephalic waveform. About $25 \%$ of all subjects became drowsy and were engaged in a brief conversation, and about $5 \%$ of the subjects were removed from the booth for walks to restore wakefulness.

The subject was seated in a reclining chair inside an electrically shielded, sound-treated room. A shielded insert earphone (Etymotic, model ER-3A) was placed in the left or right external ear canal depending upon whether the subject's number was even or odd. The order of data collection was ABR, MLR, LLR. The earphone then was moved to the second ear, electrode placement and impedance checked, encouragement given the subject, and data collected for the second ear in the same order as for the first.

Initially, two click levels, $35 \mathrm{~dB}$ apart, were used to obtain ABR data, but only the higher click level was used for the MLR and LLR conditions. About midway through the experiment, a preliminary examination of the data suggested that the lower click level might be more revealing for present purposes, and from there on, both click levels were used for ABR, MLR, and LLR. Accordingly, the number of subjects $(N)$ contributing to a condition varied.

The acoustic signals were created by exciting the earphone with a 100- $\mu$ s electrical pulse of negative polarity. The signals were presented either 35 or 70 $\mathrm{dB}$ above the average absolute sensitivity for these clicks, as measured in 20 listeners with normal hearing. The pulses were presented as a continuous train at a repetition rate that was different for the different AEPs. For ABR, MLR, and LLR, the rates were 18.1, 7.1, and 1.1 clicks per second, respectively. For each click presentation, data were collected during a "sweep" that began prior to click presentation and extended past click presentation by the same time 
interval. The durations of the sweeps were 20, 120, and $700 \mathrm{~ms}$ for ABR, MLR, and LLR, respectively.

Two channels of data were acquired simultaneously, one from each side of the head. The lead from the electrode on the vertex was split and routed to the noninverting channels of two identical physiological amplifiers, the leads from the left and right earlobes provided the input for the inverting channel of each amplifier, and the lead from the forehead electrode served as the common ground for both amplifiers. The electrode impedance was less than $5 \mathrm{k} \Omega$ and did not differ across electrodes by more than $2 \mathrm{k} \Omega$. The scalp-recorded potential was differentially amplified (gain $=200,000)$ and band-pass filtered (filter rejection rate $=-6 \mathrm{~dB}$ /octave). The pass band of the filter was $0.1-3 \mathrm{kHz}$ for the ABR, $10-300 \mathrm{~Hz}$ for the MLR, and $1-30 \mathrm{~Hz}$ for the LLR. The amplifiers were calibrated weekly, and the amplifier channels were alternated for successive subjects to control for any slight differences in gain. During each sweep, the voltage waveform in each channel was digitized using a 16-bit digital-to-analog converter having a sampling rate of $10 \mathrm{kHz}$ (MLR and LLR) or $50 \mathrm{kHz}$ (ABR). Any sweep having a peak voltage exceeding a predetermined criterion was discarded. The number of sweeps ultimately averaged to obtain a waveform was 4000 for ABR, 2000 for MLR, and 400 for LLR.

In fact, each sequence of sweeps yielded two waveforms, one for the electrode ipsilateral to the ear containing the earphone and one for the contralateral electrode. Thus, after data collection from both ears, four composite waveforms existed for each test condition. Latencies and amplitudes were estimated for the various waves in each of the four waveforms, and those values were averaged as follows: left ipsilateral value (latency or amplitude) with right contralateral value, and right ipsilateral value with left contralateral value. That is, estimates were obtained for events on each side of the head, irrespective of ear of stimulation. (The sole exception was ABR, wave I, for which all contralateral data were discarded on the grounds that such responses were likely to be attributable to crossconduction of the click stimulus.) In what follows, these are called two-way means; four-way means were also calculated for each subject, and these were used for most of the statistical analyses. To be included in the statistical analyses for a particular variable, a subject had to have a value of latency or amplitude for that measure from all four waveforms; accordingly, the Ns in groups varied across measures.

Four trained audiologists worked as experimenters in teams of two, alternating the various duties. These experimenters were also responsible for extracting the estimates of amplitude and latency for the relevant peaks from the stored AEP waveforms; all had at least two years experience judging AEP waveforms. All waveform scoring was done in ignorance of the sexual orientation of the subject under consideration.

Of interest here were two ABR peaks (I and V), three MLR peaks $(\mathrm{Na}, \mathrm{Nb}$, and $\mathrm{Pa})$, and four LLR peaks (N1, N2, P1, and P2), for a total of nine peaks. For each peak a measure of latency was obtained, and for peaks I, V, Na, and N1, a measure of amplitude was also obtained. Because data were collected using two click levels for some peaks, there was a total of 19 measures of latency or amplitude. Amplitudes of peaks were calculated from the initial inflection to the next inflection.

Statistical analysis consisted of unpaired $t$-tests and two-factor ANOVAs. Because the various measures were correlated to different degrees, it was difficult to know how to adjust the alpha level to compensate for the large number of statistical comparisons being made. Initially, we treat probability values of 0.05 and smaller as statistically significant and values between 0.05 and 0.10 as marginally significant; then we do a simulation that takes the various existing dependencies into account. Effect size was also calculated for the interesting pairwise comparisons between groups. Here the effect size corresponded to the difference in the two means divided by the square root of the weighted mean of their two variances. Cohen (1992) suggested that effect sizes of $0.2,0.5$, and 0.8 could be regarded as small, medium, and large, respectively, for comparisons of this sort.

Data are reported for a total of 49 heterosexual females (FHt), 34 homosexual females (FHm), 23 bisexual females (FBi), 50 heterosexual males (MHt), 45 homosexual males (MHm), and 8 bisexual males (MBi), although all subjects did not yield usable data for all measures. Not included in these totals are 15 females and 8 males excluded for failing the audiometric tests; 10 females and 3 males excluded for giving questionnaire responses that precluded their being categorized into one of our six categories of sexual orientation; 5 females and 3 males excluded for incomplete AEP data; and 39 heterosexual females, 2 homosexual females, and 9 bisexual females excluded for using oral contraceptives or contraceptive injections (McFadden 2000).

\section{RESULTS}

Visual inspection suggested that the data were typically quite similar for the homosexual females and bisexual females, and unpaired $t$-tests confirmed that these groups differed significantly on only one of the 19 measures [ABR, wave 1 , latency, 70-dB clicks; $t(49)=$ 2.11, $p=0.04$ ]. [The differences for ABR Wave-V latency at 70 and $35 \mathrm{~dB}$ were marginally significant.] 
Consequently, the data for homosexual and bisexual females were pooled for all subsequent analyses. The $N$ for bisexual males was so small that the data for the homosexual and bisexual males also were pooled, yielding four categories of sexual orientation for purposes of statistical analysis.

For each of the 19 measures, unpaired $t$-tests and effect sizes were calculated for three comparisons: heterosexual females against heterosexual males (the basic sex difference), heterosexual females against homosexual plus bisexual females, and heterosexual males against homosexual plus bisexual males, all using the four-way means. In Table 1 the $p$ values associated with those $t$-tests are shown along with the corresponding effect sizes and $N$. [The $N$ values shown do not agree perfectly with those in McFadden (2000) because a few subjects were inadvertently omitted there.] On the basis of these calculations, a subset of the 19 measures was selected for further analysis. The selected measures were those showing an effect size greater than 0.35 [midway between a small and medium effect size according to Cohen (1992)] and a significant, or marginally significant, difference between the heterosexuals and nonheterosexuals for one of the sexes. Five measures exceeded these inclusion criteria for the females and five for the males, with overlap on one measure. Those 10 instances are printed in bold typeface in Table 1 . Note that the sixth measure in the hierarchy for both males and females was not a close contender, that differences between heterosexuals and nonheterosexuals existed only for components of the ABR and MLR, not the LLR, and that most of the MLR and LLR measures did not show a basic sex difference. Inspection of the signs of the effect sizes in Table 1 will reveal that the well-known inverse relationship between latency and amplitude (Hall 1992) was obtained for wave V of the ABR, but was generally absent for the other peaks of the AEP.

Figure 1 presents the data for four of the five measures exceeding the inclusion criteria for the comparison between heterosexual and nonheterosexual females (the fifth measure is shown at the bottom of Figure 2). The data for the AEP peak with the shortest latency are shown in the bottom panel and those for the peak with the longest latency are in the top panels. The data in the top and third from the top panels of Figure 1 have a pattern similar to that seen previously for OAEs (McFadden and Pasanen 1998, 1999). Namely, there was a substantial basic sex difference between the heterosexual males and females, and the means for the nonheterosexual females were shifted away from those of the heterosexual females in the male direction. That is, these panels illustrate an apparent masculinization of the auditory systems of the homosexual and bisexual females. In contrast, the data in the two other panels of Figure 1 do not contain a basic sex difference; nevertheless, the means for the homosexual and bisexual females were shifted away from those of the heterosexual females.

Figure 2 contains the five measures exceeding the inclusion criteria for the comparison between heterosexual and nonheterosexual males. (The bottom panel of Figure 2 contains the one measure meeting the inclusion criteria for both the males and the females; see bold type in Table 1.) For the measures shown in the second and third panels from the top in Figure 2, there was no basic sex difference between heterosexual males and heterosexual females, but the means for the nonheterosexual males were shifted away from those of the heterosexual males. For the other three measures shown in Figure 2, there was a substantial basic sex difference, and in each case the means for the nonheterosexual males were shifted away from those of both the heterosexual males and the heterosexual females. This direction of shift leads to the nonheterosexual males appearing to be even more masculine than the heterosexual males on these AEP measures. As is discussed below, "hypermasculinization" effects of this sort have been observed previously in homosexual males (Bogaert and Hershberger 1999; Williams et al. 2000).

Noticeable in Figures 1 and 2 is the fact that the side of the brain showing the stronger or faster response varied in a complex way across sex and sexual orientation, suggesting that the auditory brains of homosexuals and heterosexuals might differ in their lateral asymmetry as well as in their strength of response to click stimuli (Reite et al. 1995; Sanders and Wright 1997; Wegesin 1998a). However, two-factor ANOVAs conducted on these data (4 categories of sexual orientation $\times 2$ sides of the head, with repeated measures on the second factor) revealed interactions that were only marginally significant, and those only for three of the nine measures shown in and Figures 1 and 2. Because of the irregular patterns of differences between the two sides of the head, the main effect for side of head was significant in only one of these nine two-factor ANOVAs [ABR, wave 1, amplitude, 70-dB clicks; $F(3,182)=7.71, p=0.006]$. Apparently, whatever factors contribute to asymmetry in AEP measures are not closely tied to the factors producing the differences in AEPs between heterosexuals and nonheterosexuals.

\section{Simulations}

Interpreting the results reported here is complicated because a large number of measures were subjected to statistical analysis, and those measures-having been collected from the same subjects-are not independent. Correlations were calculated among the five AEP measures of interest because of differences between 
TABLE 1

Effect sizes (first row) and $p$ values from a series of unpaired $t$-tests (second row; in italics) both calculated using the 4-way means from each subject. ${ }^{a}$

\begin{tabular}{|c|c|c|c|c|c|}
\hline Condition & $N^{d}$ & FHt vs. MHt ${ }^{b}$ & $\begin{array}{c}F H t \text { vs. } \\
F H m+F B i^{b}\end{array}$ & $\begin{array}{r}\mathrm{MH} \\
\mathrm{MHm}\end{array}$ & $\begin{array}{l}\text { t vs. } \\
+M B i^{b} \\
\text { to }\end{array}$ \\
\hline ABR, Wave I & $47 / 51 / 45 / 43$ & 0.12 & $0.47^{c}$ & & 0.08 \\
\hline Latency, $70 \mathrm{~dB}$ & & 0.570 & 0.024 & 0.716 & \\
\hline ABR, Wave I & $47 / 51 / 45 / 43$ & 0.59 & 0.37 & & 0.47 \\
\hline Amplitude, $70 \mathrm{~dB}$ & & 0.006 & 0.070 & 0.032 & \\
\hline ABR, Wave V & $47 / 55 / 53 / 49$ & -0.62 & 0.10 & & -0.09 \\
\hline Latency, $70 \mathrm{~dB}$ & & 0.003 & 0.605 & 0.662 & \\
\hline$A B R$, Wave $V$ & $47 / 55 / 53 / 49$ & 0.78 & -0.01 & & 0.25 \\
\hline Amplitude, $70 \mathrm{~dB}$ & & 0.0003 & 0.950 & 0.217 & \\
\hline ABR, Wave V & $47 / 53 / 52 / 46$ & -0.85 & -0.62 & & -0.21 \\
\hline Latency, $35 \mathrm{~dB}$ & & 0.0001 & 0.003 & 0.299 & \\
\hline ABR, Wave $\mathrm{V}$ & $47 / 53 / 52 / 46$ & 0.51 & 0.24 & & 0.54 \\
\hline Amplitude, $35 \mathrm{~dB}$ & & 0.017 & 0.226 & 0.009 & \\
\hline MLR, Wave Na & $48 / 55 / 50 / 46$ & -0.14 & 0.02 & & -0.65 \\
\hline Latency, $70 \mathrm{~dB}$ & & 0.486 & 0.925 & 0.002 & \\
\hline MLR, Wave Na & $46 / 55 / 50 / 46$ & -0.01 & -0.22 & & -0.03 \\
\hline Amplitude, $70 \mathrm{~dB}$ & & 0.949 & 0.279 & 0.901 & \\
\hline MLR, Wave Na & $23 / 23 / 21 / 21$ & -0.08 & 0.53 & & 0.20 \\
\hline Latency, $35 \mathrm{~dB}$ & & 0.785 & 0.078 & 0.519 & \\
\hline MLR, Wave Na & $23 / 23 / 21 / 21$ & -0.52 & -0.59 & & 0.06 \\
\hline Amplitude, $35 \mathrm{~dB}$ & & 0.093 & 0.053 & 0.860 & \\
\hline MLR, Wave Pa & $46 / 54 / 50 / 46$ & 0.02 & 0.13 & & -0.45 \\
\hline Latency, $70 \mathrm{~dB}$ & & 0.926 & 0.527 & 0.032 & \\
\hline MLR, Wave Nb & $45 / 54 / 50 / 45$ & -0.35 & -0.11 & & -0.38 \\
\hline Latency, $70 \mathrm{~dB}$ & & 0.105 & 0.581 & 0.071 & \\
\hline LLR, Wave P1 & 48/49/45/41 & -0.09 & -0.07 & & -0.04 \\
\hline Latency, $70 \mathrm{~dB}$ & & 0.685 & 0.740 & 0.855 & \\
\hline LLR, Wave N1 & $48 / 53 / 51 / 45$ & -0.23 & -0.02 & & 0.12 \\
\hline Latency, $70 \mathrm{~dB}$ & & 0.281 & 0.934 & 0.566 & \\
\hline LLR, Wave N1 & 48/53/51/45 & 0.53 & -0.04 & & -0.08 \\
\hline Amplitude, $70 \mathrm{~dB}$ & & 0.012 & 0.844 & 0.703 & \\
\hline LLR, Wave N1 & 21/23/18/18 & 0.34 & 0.08 & & -0.26 \\
\hline Latency, $35 \mathrm{~dB}$ & & 0.301 & 0.793 & 0.436 & \\
\hline LLR, Wave N1 & 21/23/18/18 & 0.18 & -0.19 & & 0.04 \\
\hline Amplitude, $35 \mathrm{~dB}$ & & 0.580 & 0.524 & 0.907 & \\
\hline LLR, Wave P2 & $48 / 53 / 51 / 45$ & 0.02 & 0.30 & & 0.21 \\
\hline Latency, $70 \mathrm{~dB}$ & & 0.929 & 0.130 & 0.305 & \\
\hline LLR, Wave N2 & $47 / 52 / 50 / 44$ & 0.47 & -0.05 & & -0.09 \\
\hline Latency, $70 \mathrm{~dB}$ & & 0.028 & 0.819 & 0.667 & \\
\hline
\end{tabular}

${ }^{a}$ Entries are two-tailed $p$ values obtained from unpaired $t$-tests comparing the two groups at the top of each column.

${ }^{b}$ Abbreviations are FHt (heterosexual females), FHm (homosexual females), FBi (bisexual females), MHm (homosexual males), MBi (bisexual males), and MHt (heterosexual males).

'Entries in bold designate those AEP measures presented in the figures and used for further statistical analysis.

${ }^{d}$ The $\mathrm{Ns}$ shown are for $\mathrm{FHt}, \mathrm{FHm}+\mathrm{FBi}, \mathrm{MHm}+\mathrm{MBi}, \mathrm{MHt}$, respectively.

the heterosexual and nonheterosexual females, and for the five measures of interest because of differences between the heterosexual and nonheterosexual males (only the heterosexual females or males, respectively, were included for those calculations). For the five AEP measures of interest for the females, six of the ten possible correlations obtained were smaller than 0.3 , and the range was 0.045 (for $\mathrm{ABR}$ wave $\mathrm{V}$ latency at $35 \mathrm{~dB}$ with MLR wave Na amplitude at $35 \mathrm{~dB}$ ) to-0.504 (for $\mathrm{ABR}$ wave $\mathrm{V}$ amplitude at $70 \mathrm{~dB}$ with $\mathrm{ABR}$ wave $\mathrm{V}$ latency at $35 \mathrm{~dB}$ ). For the five measures of interest for the males, eight of the ten possible correlations obtained were smaller than 0.3 , and the range was0.012 (for $\mathrm{ABR}$ wave 5 amplitude at $35 \mathrm{~dB}$ with MLR wave $\mathrm{Na}$ latency at $70 \mathrm{~dB}$ ) to 0.483 (for MLR wave $\mathrm{Na}$ latency at $70 \mathrm{~dB}$ with MLR wave Pa latency at $70 \mathrm{~dB}$ ).

In an attempt to assess the probability of obtaining the results reported here, a simulation was performed that takes into account the various dependencies in the data. [Simulations of this type were brought to our attention by Professor J.C. Loehlin for use in a previous report (McFadden 2000).] Separate simulations were done for the males and females. For the female simulation, random samples of 49 subjects (the number of 


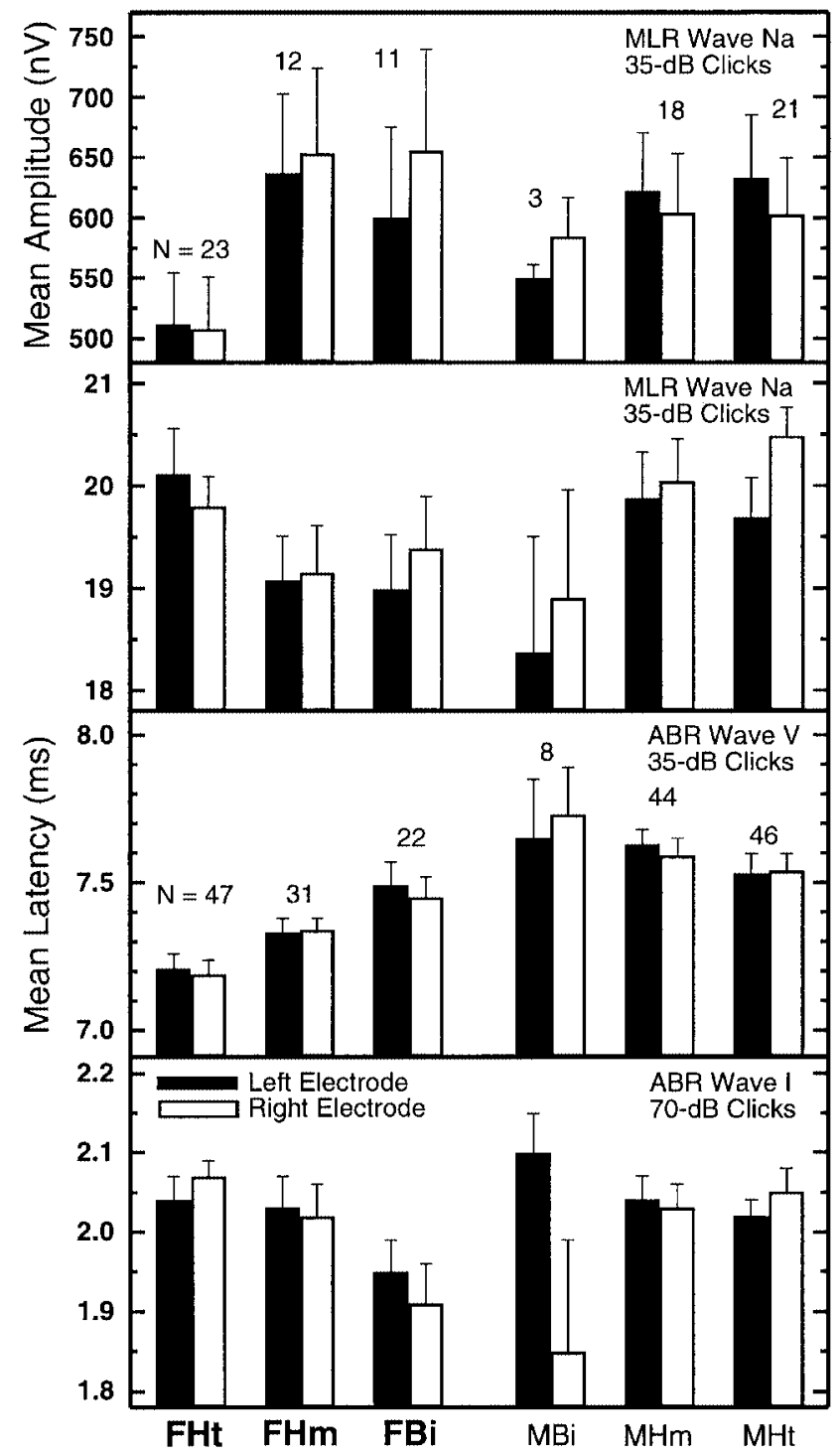

FIG. 1. Averaged auditory evoked potential data for subjects in six categories of sexual orientation. The four measures shown are ones exhibiting differences between heterosexual and nonheterosexual females. The solid and open bars denote the data collected from the electrodes over the left and right hemispheres of the brain, respectively; each bar represents averages across the ipsilateral and contralateral presentations of the clicks (the two-way means). Error flags indicate one standard error of the mean. For exact $N$ values, see Table 1.

heterosexual females in this study) were drawn from the pool of 106 (all the female subjects in the study). For each of the 19 AEP measures shown in Table 1, an effect size and an unpaired $t$-test were calculated between the group sampled and the remainder of the female subjects. The absolute values of the effect sizes of all measures achieving both an effect size of 0.35 and a $p$ value smaller than 0.1 were summed, and the result was compared with the sum of the absolute values of the effect sizes actually obtained for the five

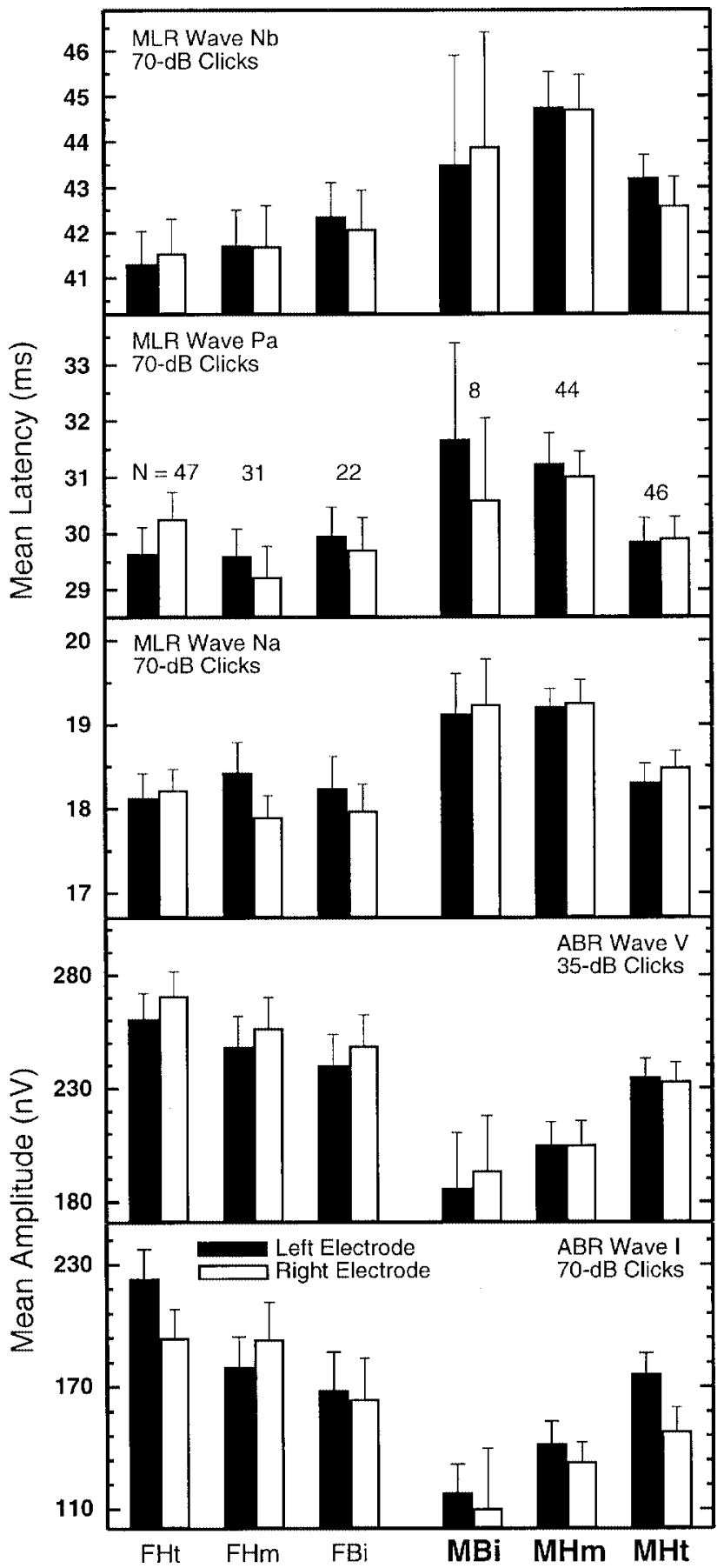

FIG. 2. Similar to Figure 1 except these are the five AEP measures showing differences between heterosexual and nonheterosexual males. (The measure shown in the bottom panel also contains a difference for the females.)

measures meeting those same criteria for the difference between heterosexual females and nonheterosexual females in this study. This procedure was implemented 10,000 times and a tally kept of the number of times the sum of the effect sizes for the simulation equaled or exceeded the sum of the effect sizes actually obtained in the data. This occurred 305 times 
in the 10,000 samples for females, corresponding to an implied probability of 0.03 for the overall pattern of results obtained. When the same procedure was implemented for the male subjects, the sum of the effect sizes actually obtained for the five measures meeting the dual criteria was equaled or exceeded by the sum of the effect sizes in the samples 491 times, corresponding to an implied probability of 0.049 .

A strength of simulations of this sort is that whatever dependencies exist between and among measures in the obtained data also exist in the simulation samples, meaning that such simulations provide a relatively unbiased estimate of the likelihood of obtaining the magnitudes of results observed. It appears safe to conclude that the probability of obtaining results of the magnitude reported here sheerly by chance was small. [In fact, the sampling procedure used guaranteed that each simulation sample would contain the appropriate number of male or female subjects having scores on the four small- $N$ measures (see Table 1). The sum of the effect sizes was used instead of the mean to offset the effect of different numbers of measures exceeding the two criteria on different samples.]

\section{DISCUSSION}

A number of diverse findings imply that the bodies and brains of homosexual males and females differ subtly in structure and function from those of their heterosexual counterparts (e.g., Perkins 1981; Sanders and Ross-Field 1986; Lindesay 1987; Gladue et al. 1990; McCormick et al. 1990; Swaab and Hofman 1990; Bailey and Pillard 1991; Levay 1991; McCormick and Witelson 1991; Allen and Gorski 1992; Bailey et al. 1993; Hall and Kimura 1994; McCormick and Witelson 1994; Scamvougeras et al. 1994; Reite et al. 1995; Blanchard and Bogaert 1996; Sanders and Wright 1997; Wegesin 1998a, b; McFadden and Pasanen, 1998, 1999; Hamer 1999; Rice et al. 1999; Singh et al. 1999; Bogaert and Hershberger 1999; Williams et al. 2000). To this growing list of physiological concomitants to homosexuality can now be added the present findings about AEPs. For both females and males, the latencies and/or amplitudes of certain AEP peaks exhibited differences between heterosexuals and nonheterosexuals, although the specific measures showing these differences were not the same for females and males. For neither sex were the differences between heterosexuals and nonheterosexuals particularly large, but simulations revealed that the probability was small of obtaining by chance differences of the magnitude observed.

For both females and males, some of the measures showing differences between heterosexuals and nonheterosexuals exhibited no basic sex difference between the heterosexual females and the heterosexual males. Those measures provide little insight into the mechanisms underlying the differences between heterosexuals and nonheterosexuals. However, for both females and males, some of the measures showing differences between heterosexuals and nonheterosexuals did exhibit a basic sex difference. For those measures, the data for the homosexual and bisexual females were shifted toward the data of the males, in accord with other auditory findings (McFadden and Pasanen 1998, 1999). This direction of shift suggests that a masculinization process of some sort is responsible for the differences observed. For the males, those measures showing a basic sex difference were shifted away from both the heterosexual males and females, thus suggesting the action of a hypermasculinization process of some sort.

Because the cochleas of nonheterosexual females were previously shown to be masculinized (McFadden and Pasanen 1998, 1999), it is logically possible that the present AEP differences between heterosexual and nonheterosexual females are simply an extension of that cochlear difference. That is, logically, the AEP differences might simply represent a "carry-through" of a difference existing at an earlier stage of auditory processing. The click-evoked OAEs (CEOAEs) of nonheterosexual females are weaker than those of heterosexual females (McFadden and Pasanen 1998), implying that their cochlear amplifiers (Davis 1983) are weaker. This diminished cochlear response to the click stimulus might manifest itself as either a slowing or a diminution in the amplitude of the neural response throughout the early stages of the auditory nervous system, without those early neural stages themselves actually being different structurally or functionally in heterosexual and nonheterosexual females. Contradicting this possible interpretation is the fact that three of the five AEP measures exhibiting differences for nonheterosexual females did not exhibit a significant basic sex difference between the heterosexual males and heterosexual females, even though CEOAEs are substantially different for those two groups. If those AEP measures do not differ between groups known to have large CEOAE (cochlear) differences, then it is difficult to see how cochlear differences could be the basis for the AEP differences between heterosexual and nonheterosexual females. Further contradicting the possibility of a carry-through effect from cochlea to auditory brain is the fact that not all measures of the early peaks exhibited a masculinizing effect for the female homosexuals and bisexuals. This possibility of a carry-through effect from cochlea to brain is not relevant to the AEP differences seen for the homosexual males because their CEOAEs did not differ from those of heterosexual males (McFadden and Pasanen 1998). Accordingly, it 
appears to be appropriate to think of the present AEP results as evidence for the existence of differences in structure or function of the auditory brain for heterosexuals and nonheterosexuals of both sexes, with the involved structures apparently not being identical for the two sexes (only one AEP measure showed differences for nonheterosexuals of both sexes).

The hypermasculinization effects observed here for the homosexual males may be unexpected and counterintuitive, but they are not without precedent. Bogaertand Hershberger (1999) examined various measures of penile length and circumference collected from about 4200 men and found that, for all measures, the penises of homosexual men were larger than those of heterosexual men. [It is interesting that Herman et al. (2000) reported larger penises in rhesus monkeys that had been exposed to higher than normal levels of androgens late in prenatal development.] In heterosexual females, the ratio of the length of the index finger to that of the fourth finger (2D:4D) is close to 1.0 for the right hand but is smaller than 1.0 for the left hand (e.g., Williams et al. 2000). In heterosexual males, the index finger is shorter than the fourth finger, but there is no asymmetry in the 2D:4D ratios in the two hands like that seen in heterosexual females. In homosexual males, however, there is an asymmetry; the 2D:4D ratio for the right hand is smaller than that of the left (Williams et al. 2000). Because this asymmetry is opposite to that in heterosexual females and because heterosexual males are not asymmetric, the finger-length data for the homosexual males can be interpreted as a hypermasculinization. Finally, Lindesay (1987) has noted that the high incidence of not-consistently-right-handers in homosexual males can be interpreted as a hypermasculinization.

It is logically possible that there is something in the lifestyles of nonheterosexual males and females that is responsible for our finding these differences in their AEPs. A number of plausible lifestyle differences (such as differential hearing loss induced by exposure to intense noise or ototoxic drugs) were ruled out as major contributors to the differences seen in the OAE data (McFadden and Pasanen 1999), and precautions were taken here as well against those factors (see Methods). Furthermore, some evidence suggests that even rather large, undetected hearing losses should have had minimal effect on the present AEP measures (Jerger and Johnson 1988). Even so, it is never possible to rule out completely all possible lifestyle differences with the fundamentally correlational research designs that are available to investigators interested in this aspect of human behavior. With that said, it is possible to account for the present results with an explanation that does not appeal to lifestyle differences and that has the virtues of being parsimonious, plausible, and in accord with numerous facts of prenatal development and sexual differentiation.

As described in detail in McFadden and Pasanen (1998, 1999), a single parsimonious explanation appears to account for a number of facts about OAEstheir greater strength and number in females than males, in both adults (Talmadge et al. 1993; McFadden and Pasanen 1998, 1999) and children (Burns et al. 1992; Norton 1992); their greater strength and number in females having male cotwins (McFadden 1993; McFadden et al. 1996); and their greater strength and number in heterosexual than in nonheterosexual females (McFadden and Pasanen 1998, 1999). That explanation suggests that the cochlear amplifiers (Davis 1983) are weakened by exposure to androgens during prenatal development and, having been weakened, the cochlear amplifiers produce fewer and weaker OAEs. Because, for females, the basic pattern of AEP results was similar to that for the OAE results, it is not unreasonable to extend that same explanation to the present findings for females. Accordingly, the masculinization seen in the AEP data for nonheterosexual females would be attributed to their having been exposed to higher-than-normal levels of androgens (for females) at some point during prenatal development, and, as a result, their cochleas, certain sites in the auditory nervous system contributing to AEPs, and whatever brain site(s) are responsible for sexual orientation in females, were masculinized. There is nothing revolutionary about such a proposal; there is a long history of discussion about the possibility that homosexuality is determined by biological factors, including atypical exposure to androgens during prenatal development (Phoenix et al. 1959; Ellis and Ames 1987; Byne and Parsons 1993). The fact that the AEPs of infants exhibit the same sex differencesas those of adults (Chiarenza et al. 1988) strengthens the possibility that the proposed overexposure to androgens occurs prenatally; if those basic sex differences are attributable to degree of androgen exposure (which seems highly likely), then it does not seem implausible that prenatal overexposure to androgen could be responsible for the masculinized auditory systems of nonheterosexual females.

If the androgen-exposure explanation is extended to the AEP results for males, then the implication is that homosexual males also were exposed to higherthan-normal levels of androgens (for males) at some point in prenatal development. At first hearing, this explanation appears to be in the wrong direction because homosexual males exhibit the prototypically female choice in sex partners, not a hypermasculine choice. One way to reconcile the apparent hypermasculinization of the AEP measures with the female-like choice of sex partner is to assume that the brain structures responsible for the AEP effects reported here did 
in fact receive higher-than-normal levels of androgen exposure, but that the brain structures responsible for sexual orientation in males received a less-than-normal exposure, resulting in an absence of masculinization of the latter structures. A second, and seemingly simpler, approach is to assume a nonmonotonic response to androgen level, whereby a little androgen masculinizes a trait from its default female state, and more androgen returns the trait to its default state. Examples of nonmonotonic effects of this sort exist in the animal literature. For example (from Clark et al. 1996), gerbils exhibit a species-typical tripodal stance in which only one forepaw contacts the ground. Male gerbils are more likely to rest on their right forepaw and females are more likely to rest on their left forepaw. However, male gerbils injected with low levels of testosterone soon after birth exhibit the female-typical stance (resting on the left forepaw) more often than do control animals. That is, an excess of testosterone apparently leads to a nonmonotonicity that pushes these animals back toward the female prototype, at least for this behavior. Accordingly, one could speculate that homosexual males get exposed to higher-than-normal androgen levels at some point early in development, and this exposure hypermasculinizes whatever brain structures are involved in producing the AEP effects reported here [as well as other structures (Bogaert and Hershberger 1999; Williams et al. 2000)], and reverses the masculinization of whatever brain structures are responsible for sexual orientation in males. Unexplained in this account is why there are hypermasculinization and nonmonotonic effects for some structures and traits and not for others (such as OAEs). The answer may lie in different magnitudes and/or timing of androgen exposure in different brain centers, differential local sensitivities to the androgen levels present at a given time, differential conversion of testosterone into estradiol, and other local factors. [Note that this second version of the androgen-exposure explanation appears to fit more parsimoniously with the existence of hypermasculinization in the relative lengths of the fingers (Williams et al. 2000) and the size of the penis (Bogaert and Hershberger 1999) in homosexual males, and with the increase in penis size in rhesus monkeys exposed to high androgen levels late in prenatal development (Herman et al. 2000).]

We note in passing that, for convenience, degree of androgen exposure is treated here as if it were a simple matter of amount present or available. However, it is also possible that the amounts available are the same in heterosexual and nonheterosexual subjects, but that, for whatever reason, some subjects, or some brain sites, are hypersensitive to the androgen levels present during some stage(s) in early development. This possibility is intuitively attractive because it emphasizes spatially and temporally localized effects in the brain, an emphasis that appears to be necessary to account for the overwhelming similarity of physiology and behavior in heterosexuals and nonheterosexuals. Furthermore, it is logically possible that antiestrogenic mechanisms are involved, also or instead of androgenic mechanisms, and in one or both sexes. The term defeminization may have had descriptive power equal to that of masculinization for the data of the nonheterosexual females, but the former term is typically reserved for discussions of complex, multiplydetermined behaviors (Whalen 1974), not for simple physiological measures varying along a single dimension, like the AEP measures discussed here.

Surely the most perplexing questions raised by these data are: Why, for females and males, differences were seen only for five of the 19 AEP measures examined, why those particular five measures were different, why the set of five measures was different for the two sexes, and why amplitude and latency were not both different for a particular wave. Answering those questions will require more knowledge than currently exists about the origins of the various AEP waves, the development of the auditory system, and the physiological mechanisms involved in homosexuality in the two sexes. What can be said is largely cautionary. An obvious first point to make is that minor variations in the details of the AEP procedure (such as different signal levels, different numbers of sweeps, different or additional electrode placements) might have revealed differences in additional measures than those reported here. Second, it is important to realize that there almost surely is not a one-to-one correspondence between individual AEP peaks and single locations in the brain. That is, it is almost certainly incorrect to presume that the succession of waves in the AEP originates from a succession of auditory nuclei each further along the afferent chain that stretches from cochlea to cortex (Hall 1992, p. 55). Surely, the later waves do contain contributions from higher nuclei that do not contribute to the earliest waves, but the later waves also are likely to contain contributions from populations of neurons low in the chain that just happen to be activated (or reactivated) synchronously with whatever higher-level neurons are being activated.

The point is that observing a difference between heterosexuals and nonheterosexuals in the latency or amplitude of some particular AEP wave does not correspond unambiguously to a difference in structure or function in some one brain location. Rather, it is likely that multiple populations at different locations are involved, especially for the later waves. Identifying exactly which neural populations are responsible for the differences observed here is likely to be a protracted endeavor. Furthermore, it should be appreciated that even if (when) those neural populations are 
identified, it will not amount to having identified populations involved in determining sexual orientation because those identified neurons will be squarely in the auditory system and will be responsible only for processing auditory input. Whatever differences are eventually found to exist in those populations of auditory neurons must be seen and interpreted for what they are-simply physiological concomitants to those neural differences that are actually responsible for sexual orientation and behavior. That is, our belief is that the differences we have observed in the auditory systems of heterosexuals and nonheterosexuals are themselves results of some of the same factors operating during development (possibly prenatal development) that also affected those brain locations responsible for sexual orientation. For a number of reasons, a plausible working hypothesis is that the magnitude of exposure to androgens was involved in producing both the auditory differences and the homosexuality, and additional work is planned on this hypothesis. Admittedly, the fact that the five measures showing differences for the females were generally different from the five measures showing differences for the males is interesting and does support the idea that the physiological bases for homosexuality may be different in the two sexes (e.g., Bailey et al. 1993), but the indirect correlational relationship between our auditory measures and the brain structures responsible for sexual orientation makes strong conclusions premature.

The AEP differences reported here for homosexual and bisexual males and females, in conjunction with the previous findings about OAEs (McFadden 1993; McFadden and Pasanen 1998, 1999), suggest that the auditory system has the potential to serve as a valuable supplementary tool for the study of hormonal effects on the nervous system during development. We wish to emphasize that the differences reported here, like those for OAEs (McFadden and Pasanen 1998, 1999), are average group differences and could not be used to identify reliably the sexual orientation of an individual person.

\section{ACKNOWLEDGMENTS}

We greatly appreciate the help of the following with data collection and waveform scoring: A. Harkrider, M. Dean, K. Hill, J. Marler. The following helped with recruiting and scheduling subjects and data entry and reduction: C. Scott, M. Dittrich, L. Strother, A. Phan, D. Simmons, and M. Huebner. E.G. Pasanen provided valuable assistance with the simulations; he, J. Loehlin, C. Meston, and E. Hull commented on a preliminary draft of this paper. The comments of two anonymous reviewers also improved the paper. This work was supported by research grants from the National
Institute on Deafness and other Communication Disorders (NIDCD 00153 to DM and 001415 to CAC).

\section{REFERENCES}

ALLEN LS, GORSKI RA. Sexual orientation and the size of the anterior commissure in the human brain. Proc. Natl. Acad. Sci. USA 89:7199-7202, 1992.

BAILEy JM, PillaRD RC. A genetic study of male sexual orientation. Arch. Gen. Psychiatry 48:1089-1096, 1991.

Bailey JM, Pillard RC, Neale MC, Agyei Y. Heritable factors influence sexual orientation in women. Arch. Gen. Psychiatry 50:217223, 1993.

BLANCHARD R, BOGAERT AF. Homosexuality in men and number of older brothers. Am. J. Psychiatry 153:27-31, 1996.

BogAert AF, Hershberger S. The relation between sexual orientation and penile size. Arch. Sex. Behav. 28:213-221, 1999.

Burns EM, Arehart KH, Campbell SL. Prevalence of spontaneous otoacoustic emissions in neonates. J. Acoust. Soc. Am. 91:1571$1575,1992$.

BuRns EM, CAMPBELL SL, AREHART KH. Longitudinal measurements of spontaneous otoacoustic emissions in infants. J. Acoust. Soc. Am. 95:385-394, 1994.

Byne W, PARSONS B. Human sexual orientation: The biologic theories reappraised. Arch. Gen. Psychiatry 50:228-239, 1993.

Chiarenza GA, D’ambrosio GM, Cazzullo AG. Sex and ear differences of brain-stem acoustic evoked potentials in a sample of normal full-term newborns. Normative study. Electroencephal. Clin. Neurophysiol. 71:357-366, 1988.

Clark MM, Robertson RK, GALEF BG Jr. Effects of perinatal testosterone on handedness of gerbils: Support for part of the Geschwind-Gallaburda hypothesis. Behav. Neurosci. 110:1-5, 1996.

CoHen J. A power primer. Psych. Bull. 112:155-159, 1992.

DAVIS H. An active process in cochlear mechanics. Hear. Res. 9:7990, 1983.

Don M, Ponton CW, EgGermont JJ, MASUdA A. Gender differences in cochlear response time: An explanation for gender amplitude differences in the unmasked auditory brain-stem response. J. Acoust. Soc. Am. 94:2135-2148, 1993.

Elkind-Hirsch KE, Stoner WR, Stach BA, Jerger JF. Estrogen influences auditory brainstem responses during the normal menstrual cycle. Hear. Res. 60:143-148, 1992.

Elkind-Hirsch KE, Wallace E, Malinak LR, Jerger JF. Sex hormones regulate ABR latency. Otolaryngol. Head Neck Surg. 110:46-52, 1994.

ELLIS L, AMEs MA. Neurohormonal functioning and sexual orientation: A theory of homosexuality-heterosexuality. Psych. Bull. 101:233-258, 1987.

Franklin DJ, McCoy MJ, Martin GK, Lonsbury-Martin BL. Test/ retest reliability of distortion-product and transiently evoked otoacoustic emissions. Ear Hear. 13:417-429, 1992.

Gladue BA, Beatty WW, Larson J, Staton RD. Sexual orientation and spatial ability in men and women. Psychobiol. 18:101-108, 1990.

HaLL JW III. Handbook of Auditory Evoked Responses Allyn and Bacon. Boston, 1992.

HALl JAY, KimURA D. Dermatoglyphic asymmetry and sexual orientation in men. Behav. Neurosci. 108:1203-1206, 1994.

HAMER DH. Genetics and male sexual orientation. Science 285:803a, 1999.

Herman RA, Jones B, Mann DR, Wallen K. Timing of prenatal androgen exposure: Anatomical and endocrine effects on juvenile male and female rhesus monkeys. Hormones and Behav. (in press) 2000 . 
JERGER J, JOHNSON K. Interactions of age, gender, and sensorineural hearing loss on ABR latency. Ear Hear. 9:168-176, 1988.

KEMP DT. Stimulated acoustic emissions from within the human auditory system. J. Acoust. Soc. Am. 64:1386-1391, 1978.

LEVAY S. A difference in hypothalamic structure between heterosexual and homosexual men. Science 253:1034-1037, 1991.

LiNDESAY J. Laterality shift in homosexual men. Neuropsychol. 25:965-969, 1987.

MCCORMick CM, Witelson SF. A cognitive profile of homosexual men compared to heterosexual men and women. Psychoneuroendocrinol. 16:459-473, 1991.

MCCORMiCK CM, WitelsOn SF. Functional cerebral asymmetry and sexual orientation in men and women. Behav. Neurosci. 108:525531, 1994.

MCCoRmick CM, Witelson SF, Kingstone E. Left-handedness in homosexual men and women: Neuroendocrine implications. Psychoneuroendocrinol. 5:69-76, 1990.

MCFAdDEN D. A masculinizing effect on the auditory systems of human females having male co-twins. Proc. Natl. Acad. Sci. USA. 90:11900-11904, 1993.

MCFADDEN D. Sex differences in the auditory system. Dev. Neuropsychol. 14:261-298, 1998.

MCFAdDEN D. Masculinizing effects on otoacoustic emissions and auditory evoked potentials in women using oral contraceptives. Hear. Res. 142:23-33, 2000. 10.1016/S0378-5955(00) 00002-2.

McFadden D, Loehlin JC, Pasanen EG. Additional findings on heritability and prenatal masculinization of cochlear mechanisms: Click-evoked otoacoustic emissions. Hear. Res. 97:102-119, 1996. 10.1016/0378-5955(96)00065-2.

MCFAdDEn D, PASANEn EG. Comparison of the auditory systems of heterosexuals and homosexuals: Click-evoked otoacoustic emissions. Proc. Natl. Acad. Sci. USA. 95:2709-2713, 1998.

McFadden D, PASANen EG. Spontaneous otoacoustic emissions in heterosexuals, homosexuals, and bisexuals. J. Acoust. Soc. Am. 105:2403-2413, 1999.

NORTON SJ. The effects of being a newborn on otoacoustic emissions. J. Acoust. Soc. Am. 91 (Suppl. 1):2409, 1992.

Perkins MW. Female homosexuality and body build. Arch. Sex. Behav. 10:337-345, 1981.

Phoenix CH, Goy RW, Gerall AA, Young WC. Organizing action of prenatally administered testosterone propionate on the tissues mediating mating behavior in the female guinea pig. Endocrinol. 65:369-382, 1959 .
Probst R, Lonsbury-Martin BL, Martin GK. A review of otoacoustic emissions. J. Acoust. Soc. Am. 89:2027-2067, 1991.

Reite M, Sheeder J, Richardson D, Teale P. Cerebral laterality in homosexual males: Preliminary communication using magnetoencephalography. Arch. Sex. Behav. 24:585-593, 1995.

Rice G, Risch N, Ebers G. Response. Science 285:803a, 1999.

SANDERS G, Ross-Field L. Sexual orientation and visuo-spatial ability. Brain Cognition 5:280-290, 1986.

SANDERS G, WRIGHT M. Sexual orientation differences in cerebral asymmetry and in the performance of sexually dimorphic cognitive and motor tasks. Arch. Sex. Behav. 26:463-480, 1997.

Scamyougeras a, Witelson SF, Bronskill M, Stanchev P, Black S, Cheung G, Steiner M, Buck B. Sexual orientation and anatomy of the corpus callosum. Soc. Neurosci. Abstr. 20:1425, 1994.

Singh D, VidaurRi M, Zambarano RJ, DabBs JM Jr. Lesbian erotic role identification: Behavioral, morphological, and hormonal correlates. J. Pers. Soc. Psych. 76:1035-1049, 1999.

SwaAb DF, Hofman MA. An enlarged suprachiasmatic nucleus in homosexual men. Brain Res. 537:141-148, 1990.

Talmadge CL, LONG GR, Murphy WJ, Tubis A. New off-line method for detecting spontaneous otoacoustic emissions in human subjects. Hear. Res. 71:170-182, 1993.

Thomas H, Jamison W, Hummel DD. Observation is insufficient for discovering that the surface of still water is invariantly horizontal. Science 181:173-174, 1973.

Trune DR, Mitchell C, Phillips DS. The relative importance of head size, gender and age on the auditory brainstem response. Hear. Res. 32:165-174, 1988.

VANDENBERG SG, Kuse AR. Mental rotations, a group test of threedimensional spatial visualization. Percept. Motor Skills 47:599601, 1978.

WEGESIN DJ. Event-related potentials in homosexual and heterosexual men and women: Sex-dimorphic patterns in verbal asymmetries and mental rotation. Brain Cognition 36:73-92, 1998a.

WEGESIN DJ. A neuropsychologic profile of homosexual and heterosexual men and women. Arch. Sex. Behav. 27:91-108, 1998b.

WHALEN RE. Sexual differentiation: Models, methods, and mechanisms. Friedman RC, Richart RH, Wiele RLV. Sex Differences in Behavior, Wiley, New York, 467-484, 1974.

Williams TJ, Pepitone Me, Christensen SE, Cooke BM, Huberman AD, Breedlove NJ, BreEdlove TJ, Jordan CL, BreEdLove SM. Finger length ratios and sexual orientation. Nature 404:455456, 2000. 